\title{
1 The information signature of diverging lineages
}

3 Douglas G. Moore ${ }^{1,2 *}$, Matheo Morales ${ }^{3,4}$, Sara I. Walker ${ }^{1,5}$, Greer A. Dolby,6*

4

51 The Beyond Center for Fundamental Concepts in Science, Arizona State University,

6 Tempe, AZ

72 39Alpha Research, Tempe, AZ

$8{ }^{3}$ School of Life Sciences, Arizona State University, Tempe, AZ

$9{ }^{4}$ Department of Genetics, Yale University, New Haven, CT

${ }^{5}$ School of Earth and Space Exploration, Arizona State University, Tempe, AZ

116 Center for Mechanisms of Evolution, Biodesign Institute, Arizona State University,

12 Tempe, AZ

14 Corresponding author(s):

DGM - douglas.g.moore@asu.edu

16

GAD - gadolby@asu.edu

Running title: Genomic information decomposition

Keywords: speciation, genomics, partial information decomposition, theory 


\section{INTRODUCTION}

25 The process of forming new species is the driving force behind the diversity of

26 life on Earth. Yet, we have not answered the basic question: why are species

27 unevenly distributed across taxonomic groups and geographic settings? This is

28 because we lack the means to directly compare aspects of population (lineage)

29 divergence across unrelated species because taxon-specific effects make

30 comparisons difficult or impossible. Here, we present a new solution to this

31 challenge by identifying the information signature of diverging lineages,

32 calculated using partial information decomposition (PID), under different

33 evolutionary scenarios. We show in silico how the informational decomposition

34 of genetic metrics varies over time since divergence. Calculating PID over 97,200

35 lattices reveals that the decomposed nodes of Tajima's $D, \theta_{w}$, and $\pi$ have strong

36 information signatures, while $F_{S T}$ was least useful for discriminating among

37 divergence scenarios. The presence or absence of gene flow during divergence

38 was the most detectable signature; mutation rate and effective population size

$39\left(N_{\mathrm{e}}\right)$ were also detectable whereas differences in recombination rate were not.

40 This work demonstrates that PID can reveal evolutionary patterns that are

41 minimally detectable using the raw metrics themselves; it does so by leveraging

42 the architecture of the genome and the partial redundancy of information

43 contained in genetic metrics. Our results demonstrate for the first time how to

44 directly compare characteristics of diverging populations even among distantly

45 related species, providing a foundational tool for understanding the diversity of

46 life across Earth. 
Many central themes of evolutionary biology deal with how new species differentiate from related ones, but many core questions about speciation remain unsolved. Among these are how often lineages diverge in isolation or with gene flow,

50 how often certain geologic or geographic settings promote speciation, and which

51 taxonomic groups are most affected (1). Answering these questions depends upon 52 comparing aspects of lineage (population) divergence, such as the amount of genetic 53 divergence and gene flow between populations, in different taxa. Yet, the organismal

54 traits that cause population pairs of species to diverge differently under a common set of conditions also influence the measures of population diversity and divergence (e.g., $\pi$,

$F_{S T}$ ) that we use to characterize those differences, making it difficult to compare

57 characteristics of diverging populations across distantly related species (Figure 1).

58 Some such aspects can be tackled using the site frequency spectrum (SFS; 2), but the

59 SFS has limitations because different histories can produce similar geometric 60 distributions (3).

In this study we take an altogether new approach by assessing the information signal of genetic metrics under different evolutionary scenarios. Multivariate analysis is

63 helpful when individual variables convey partial knowledge about a system of study.

64 Taking an example from the kinetic theory of ideal gasses, macroscopic observables

65 such as temperature and pressure provide information about the microscopic state of

66 the system; they each provide some information, but it is only when taken together that

67 the density of the gas can be determined solely through observation of those properties.

68 We can characterize and identify observables which have utility for answering specific 69 questions about the system under study by decomposing the information the 
70 observables provide over combinations of those observables, identifying the redundant,

71 unique and synergistic contributions of each observable. Along this vein, we chose to

72 decompose the multivariate information signal of different combinations of genetic

73 metrics calculated on datasets simulated under a suite of evolutionary scenarios. Using

74 the architecture of the genome, this information signal is effectively standardized within

75 a dataset making comparison across species possible.

DNA is an information storage molecule because it encodes directions for the

77 formation of proteins and those directions are heritable across generations. Information

78 processing across levels of biological organization is also thought to be a fundamental,

79 measurable characteristic that sets living systems apart from nonliving systems (4). It

80 may therefore be appropriate that information theoretic measurements reveal higher-

81 order patterns about biological evolution, which fundamentally based on the context-

82 dependency and patterned cross-generational transmission of genetic information

83 (DNA) (5).

Claude Shannon introduced mutual information in 1948 to quantify the degree to which observations of some random variable reduces the observer's uncertainty about another variable (6). Mutual information and its related information-theoretic quantities

87 have been applied in biology (7-10), machine learning and data science (11-13), 88 complex systems theory (14-16), and ecohydrology (17). As with linear measures of 89 correlation, mutual information can be generalized to a multivariate setting where total 90 correlation (18), dual total correlation (19) and interaction information (20) are

91 exemplars. We calculated the PID formalized by Williams and Beer (21) over eight 92 common genetic metrics applied to 'genic' and 'intergenic' regions of genetic data 
93 simulated for diverging populations. PID decomposes the total correlation between a

94 target variable and a collection of source variables over a lattice (Figure 1E). The nodes

95 of the lattice represent redundant, unique and synergistic contributions of combinations

96 of the source variables, and each combination yields a non-negative information

97 contribution.

98 Application of PID to genomic data here (Figure 1) is based on the premise that

99 how information about evolutionary processes is encoded across genomic elements

100 (e.g., genes), and how that information decomposes over genetic metrics (e.g., FST),

101 provides information about both the evolutionary and organismal context of that

102 sequence (Figure 1B). Consider populations diverging without gene flow (i.e.

103 reproduction). We expect these populations to accrue divergence in intergenic

104 (noncoding) elements of the genome faster than coding elements, and this discrepancy

105 would be higher in genetically isolated populations than if the populations had some

106 level of gene flow (22). To understand the information signature of genetic metrics

107 under different evolutionary settings, we simulated $60 \mathrm{kbp}$ of sequence data for 108 individuals in each of two populations under 24 evolutionary scenarios. Evolutionary 109 scenarios varied mutation rate $(\mu)$, recombination rate $(r)$, effective population size $\left(N_{e}\right)$, 110 and gene flow (M). We sampled genomes of 10 individuals per population at 10

111 timepoints per simulation to obtain a timeseries signature during divergence. Simulated 112 genomes contained 10 'intergenic' regions that evolved unconstrained and 10 'genic' 113 regions that evolved under purifying selection where mutations that changed the amino 114 acid from the ancestral state penalized the individual's fitness (see methods). We 115 simulated 100 replicates per scenario which were concatenated and used to calculate 
116 eight genetic metrics ( $F_{S T}, D_{a}$, Tajima's $D, \theta_{W}, \pi, S$, Fu's $F S$, and $D_{X Y}$ ) per genomic

117 region. For each set of genetic metrics, we calculated PID over lattices constructed from

118 combinations of up to four variables using genic/intergenic as the target variable.

119 Results yielded 97,200 PID lattices that contained 7,648,800 nodes in total. The results

120 were vectorized over timepoints to determine how the information of nodes within

121 lattices varied with time since divergence and we grouped these into temporal patterns

122 using k-means clustering. Results for the long time series ( $10^{5}$ generations) showed that

123 nodes within decomposed lattices exhibited partial information patterns that fell into

124 seven timeseries classes (Figure 2B). Most nodes were invariable $(N=302,207)$, while

125 some reliably decreased (Class $2, \mathrm{~N}=196$ ) or increased (Class $3, \mathrm{~N}=128$ ). Complex time

126 signatures are in SI (Figure S1).

We find that information signatures depended almost entirely on the

128 informativeness of a few nodes representing key genetic metrics and not on the set of

129 variables being decomposed (Figures 2a, 3, S2, S3). The only exception to this pattern

130 was Tajima's D, which increases in informativeness over time in the absence of gene

131 flow (Figure 3c). When included among the variables, Tajima's D redistributed

132 information over the lattice, making other nodes more informative even though Tajima's

133 D itself was rarely (if ever) part of the node with an informative time signature (class 2 or

1343 ) or an ability to discriminate among scenarios (e.g., Figure 3d). The variables lending

135 greatest informativeness (i.e. ability to discriminate among evolutionary scenarios) were

136 nucleotide diversity $(\pi)$ and Watterson's estimator $\left(\theta_{w}\right)$ (Tajima's $D$ was influential for

137 the reason described). The types of nodes with greatest discrimination potential were

138 either unique or redundant; synergistic nodes were never discriminatory among 
evolutionary scenarios (Figure 3). We interpret this to be due to the redundancy or nonindependence of the genetic metrics and not a behavior of PID itself.

Our exploration of evolutionary parameter space $\left(N_{e}, \mathrm{M}, \mathrm{r}\right.$, and $\left.\mu\right)$ reveals the

our simulations. However, sampling more rates of gene flow could further resolve this pattern. The variable most able to recover the gene flow signal is $\pi$ while Tajima's $D$ and $\mathrm{F}_{\mathrm{ST}}$ reflect the presence/absence of gene flow only at higher mutation rates (Figure S4). Mutation rate is recovered to a lesser extent than the presence of gene flow and is reflected best by Fu's FS. Segregating sites $(\mathrm{S})$ and Watterson's estimator $(\theta \mathrm{w})$ reflect differences in mutation rate but are also influenced by presence/absence of gene flow (Figure S5). S and $\theta_{\mathrm{w}}$ yielded identical results (Figure S5); this is expected because $\theta_{\mathrm{w}}$ is calculated by correcting $S$ for the number of samples observed which was consistent 153 across our simulations. This nonetheless demonstrates PID's consistency to record 154 equivalent patterns despite calculation over raw values of different scales. If we varied sample size, these metrics might no longer produce identical decompositions.

No nodes effectively discriminated recombination rate, which could be because

157 our metrics did not reflect patterns of genomic linkage. We speculate that decomposing 158 alternative genetic metrics could recover a difference in recombination rates. For 159 differences in $N_{e}$ (Figure S6), the magnitude of partial information was slightly inflated 160 under smaller effective population sizes (Figure 3). It is possible that additional targeted 161 exploration of 'node-space' with a focus on discriminating among $N_{e}$ could uncover a 
more useful decomposition.

We assessed the reproducibility of PID results by selecting four scenarios $(M=0$, $\left.\mu=2.5 \times 10^{-7}, \quad N_{e} \in\{500,1000\}, \quad r \in\left\{0,10^{-7}\right\}\right)$ and simulating 300 additional genetic datasets in batches of 100 per scenario (Table S2). As done previously, the eight metrics were calculated, their results concatenated per batch, and PID computed independently on the three new batches of simulations. To assess technical variance, lattices across the four batches (the one original batch and three additional replicate batches) were compared using mean-absolute-difference (MAD). Results showed the MAD between any pair of lattices never exceeded $1 e^{-4}$ bits, which is orders of magnitude below the differences we observe between evolutionary scenarios. In other words, the technical variance among genetic simulations and genomic PIDs was exceedingly small.

After as few as $10^{4}$ generations, many of the PID nodes exhibited partial information near the theoretical maximum of 1 bit. However, it is expected that before divergence begins that all nodes have negligible partial information because the populations start out from a single sequence. To confirm this, we re-simulated the first $10^{4}$ generations and sampled at a higher frequency (every $10^{3}$ generations) with the expectation that the nodes exhibiting non-zero partial information would rapidly depart from 0 bit. Results are consistent with the assumption that the information content begins at 0 bit; results show it becomes nonzero following the first generation. There is slightly less stability in the early generations evidenced by the greater number of time series clusters compared to the $10^{5}$-generation dataset ( $N=20$ vs $N=7$ groups, respectively; Figure S1 vs. S7). With additional work, it is possible that this latency 
period could be leveraged to measure aspects of very early divergence ( $\$ 1000$ generations, Figure S8). Aside from slightly reduced power in early generations to

187 discriminate among evolutionary scenarios, the information patterns stabilize once a critical threshold is met (Figure $3 \mathrm{~A}$ ). This reliability-of-pattern is favorable for the applicability of PID to genomic datasets of wild populations in which age-of-divergence dates can be reasonably presumed to be $\gtrsim 3000$ generations.

behavior of information decomposition and its application to genetic metrics and evolution. First, it is surprising that $\mathrm{F}_{\mathrm{ST}}$, which is one of the most broadly used and

194 interpretable statistics in evolution, was the least informative when decomposed, while more fundamental measures of diversity $\left(\pi, S\right.$ or $\left.\theta_{w}\right)$ drove information patterns. Also, key variables determined information patterns rather than the combination of variables

197 in the decomposition (the variable set). This finding is important because computing PID 198 on a large number of variables is costly and difficult to interpret; it suggests that small 199 lattices with a few key variables are sufficient for many purposes, and new applications 200 of PID should assess patterns within small decompositions first. Likewise, we were 201 surprised to find that the unique and redundant nodes far outweighed information in synergistic nodes. We interpret this to be a property of the genetic metrics analyzed, 203 particularly their non-independence under different evolutionary settings. If, unlike other 204 multivariate statistics, PID can leverage interpretational power from the 205 nonindependence of variables, that may be its biggest strength. It is possible that 206 synergistic terms could gain power under more evolutionarily complex scenarios, such 207 as reduced gene flow from a physical barrier in combination with differential adaptation, 
208

209

210

211

212

213

214

215

216

217

218

219

220

221

222

223

224

225

226

227

228

229

230 Genetic simulations \& evolutionary analysis population are of interest.

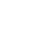

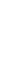

which remains a stumbling block for analyses using the SFS (24).

A final, related discovery was that combinations of variables exhibited "additive" discriminatory behavior. As an example, the single variable decomposition [S] displayed sensitivity to both $M$ (presence/absence) and $\mu$ (Figure 4a). The single variable decomposition of [D] reflected primarily presence or absence of gene flow (M, Figure 3c). The two-variable decomposition [S,D] presents a simple combination of [S] and [D], with a primary signal of gene flow/no gene flow (present in both [S] and [D]) and a secondary signal of $\mu$ (present in [S]). The discovery of additive behavior among variables should help biologists perform a targeted search of 'decomposition-space' for lattices that will discriminate between biological parameters of interest. In theory, choice of metrics to decompose can be optimized based on which properties of a diverging

In summary, answering many outstanding questions in speciation and macroevolution depends on our ability to compare characteristics of diverging lineages across diverse organisms. We outline a new information theoretic approach to do this which leverages the architecture of the genome and redundancy of well-known genetic metrics. Application of PID here shows the presence/absence of gene flow is easily detectable by several decompositions. But more importantly, PID is a new class of tool that can be applied to other variables and the large decomposition-space can be mined for nodes targeted to specific evolutionary characteristics and timepoints.

MATERIALS \& METHODS 
231 We simulated population divergence scenarios in SLiM v3.3.2 (25) using house scripts

232 that automated the parameter generation, simulations, and molecular statistic

233 calculations with EggLib (Evolutionary Genetics and Genomics Library) v3.0.0b21 (26).

234 The automated pipeline was written in python, Jinja, linux, and Eidos and allows for

235 parallelizing SLiM simulations to be run in batch on high performance computing

236 clusters (available at https://github.com/mmoral31/SLiM pipeline); it processes

237 intermediate files, and calculates diversity statistics. All simulations started with a

238 random seed and initial genome sequence of 60,000 nucleotides divided into ten

239 "coding" and ten "noncoding" regions of 3,000 nucleotides each to simulate a small

240 eukaryotic genome. The "coding" and "noncoding" regions were composed of a random

241 assortment of 1,000 codons (excluding stop codons) and 3,000 nucleotides,

242 respectively. We simulated data under all combinations of the following four parameters:

243 recombination rate $r \in\left\{0,10^{-7}\right\}$, mutation rate $\mu \in\left\{2.5 \times 10^{-7}, 2.5 \times 10^{-6}\right\}$, symmetrical

244 gene flow $M \in\{0.0,0.01,0.10\}$, and effective population size $N_{e} \in\{500,1000\}$ for a total of

24524 scenarios (Table S1). To simulate purifying selection on coding regions and calculate

246 fitness, in SLiM we translated and compared the coding regions for each individual per

247 generation to its initial (ancestral) amino acid sequence. The individual was assigned a

248 reduced fitness of 0.9 based on one amino acid mismatch and 0.5 for two or more

249 mismatches. Simulations were run for 100,000 generations and VCF (Variant Call

250 Format) files were generated every 10,000 generations; each parameter combination

251 was replicated 100 times, yielding 2,400 VCF files that were then concatenated for 252 genetic calculations.

253 To calculate genetic metrics, we used EggLib (Evolutionary Genetics and 
254 Genomics Library) on the concatenated VCFs generated by SLiM. The house script

255 calculated eight statistics: Fst, Da, Tajima's D, $\theta_{w}, \pi, S$, Fu's FS, and Dxy in each 3-kb

256 coding and noncoding region individually (i.e. window size and step size of $3 \mathrm{~kb}$; Table

257 S2). Whether the region was 'coding' or 'noncoding' was recorded in the output for each

258 window. These statistics were then passed into Imogen.jl for PID calculations.

$\underline{\text { Technical variation \& early divergence simulations }}$

To assess technical variance of simulations and its effect on PID calculations, we

ran an additional 300 replicates (for a total of $\mathrm{N}=400$ per parameter combination) for a

subset of parameters: no gene flow, mutation rate $=2.5 \times 10^{-7}$, with both population

sizes of 500 and 100, and with and without recombination (Table S2). To confirm the

theoretical assumption that the genetic metrics hold no information at the immediate

onset of divergence, and to test the reliability of decompositions during early-

267 divergence, we ran another set of simulations focusing on early time-series sampling.

268 For this we re-ran all 24 scenarios for 10,000 generations and sampled every 1,000

269 generations, with another 100 replicates per scenario (Table S2). The VCFs were

270 generated at generation two instead of generation one due to software limitations. This

271 set of data also consisted of 2,400 VCF files that were concatenated for genetic

272 analysis.

273

274 Partial information decomposition

275 Implementation \& running of analyses

276 The preceding analysis provided a sequence of values for each genetic metric along 
277 with whether or not the associated 3-kb region was a coding or noncoding region. In

278 other words, over the length of the genome, we had a binary vector $\vec{G}$ representing

279 whether or not a given region is coding, and eight vectors $\overrightarrow{d_{l}}-$ one for each evolutionary

280 statistic estimated in the region. We treated these as sequences of observations of

281 random variables $G$ and $d_{i}$ which allowed us to ask how the information about whether a

282 given region is coding decomposes over combinations of the evolutionary statistics

283 using the PID formalism. However, in general the $\overrightarrow{d_{l}}$ are real-valued, and since the

284 Williams and Beer PID formalism is limited to discrete-valued data, we performed a

285 discretization process on each $\overrightarrow{d_{l}}$. For the sake of simplicity and to reduce systematic

286 errors due to the short genome lengths $(60,000 \mathrm{bp})$, we opted to use a "mean-threshold"

287 binning scheme: the jth value of $\overrightarrow{d_{l}}$, written here as $d_{j i}$, was replaced with a 1 if it was

288 greater than the average of all values in $\overrightarrow{d_{l}}$, and 0 otherwise. This yielded a set of eight

289 binary vectors $\overrightarrow{D_{l}}$. PID could then be applied to the resulting vectors $\vec{G}$ and $\overrightarrow{D_{l}}$. In this

290 process, the multivariate mutual information between $\vec{G}$ and $\overrightarrow{D_{l}}$ was estimated and

291 subsequently decomposed into a sum of non-negative terms, roughly describing the

292 degree to which combinations of the variables $\overrightarrow{D_{l}}$ provided unique, redundant, and

293 synergistic information about whether or not a given region is coding. Due to 294 computational limitations of PID, the process can only be applied to at most five of the 295 evolutionary statistics at time. In this work, we limited calculations to at most 296 combinations of four statistics considered simultaneously to limit computation time and 297 keep the amount of data generated manageable. We carried out the computation for all 298 lattices with between one and four of the genetic metrics as source variables and the 299 region classification $\vec{G}$ as the target to yield 162 PID lattices per simulation per sampled 
generation for a total of 97,200 lattices containing $7,648,800$ nodes in total.

Information clusters \& informative metrics

303 For each node in each lattice per simulation, we created a vector of the partial

304 information $(\Pi)$ for that node over time. Many of the resulting curves had similar 305 temporal dynamics but started out with a different initial value. To account for this, we 306 shifted each vector by subtracting the zeroth element, element-wise, to make all vectors 307 start at the same zero value. To reduce the 764,880 vectors into classes of information 308 signatures over time, we performed k-means clustering based on Euclidean norm 309 distance in the 10-dimensional space formed by the 10 sampled timepoints. We used 310 the "elbow method" to identify a reasonable number of clusters for the k-means 311 analysis. That is, we plotted the explained variance against the number of clusters for a 312 range of values of $k$, and selected $k$ at or near a prominent elbow (bend) in the curve.

313 Based on this method, we chose a 7-means clustering to reduce the number of patterns 314 on which to conduct further analyses without oversimplifying the diversity of results.

Limitations

317 We note several aspects of this analysis that could affect the results obtained. First, the 318 genomes simulated were small $(60 \mathrm{kbp})$ relative to the gigabasepair-size genome of 319 vertebrates, for example. For simplicity, simulations did not incorporate standing 320 variation in the population, which is a simplification of reality. Further, certain technical 321 aspects of the Williams and Beer formalization of PID, such as the lack of localizability, 
$(27,28)$ made progress in addressing these points, and have arguably developed notions of information decomposition that are easier to interpret (which may be important for the future of PID for biological applications). The choice to use Williams and Beer's formalism necessitated a binning procedure on the computed evolutionary quantities before analysis, and the particular method of binning can have effects on the resulting decompositions. It would be worth considering different discretization methods (i.e. 29-31) to assess the sensitivity of the results presented in this work.

\section{ACKNOWLEDGEMENTS}

We thank the Baja GeoGenomics consortium for useful feedback and discussions and Kenro Kusumi for support.

DATA AVAILABILITY

Code is available on Github: https://github.com/elife-asu/gPID and https://github.com/mmoral31/SLiM pipeline

All data files will be made available upon publication.

\section{AUTHOR CONTRIBUTIONS}

DGM and GAD conceived of this project; DGM, SIW, and GAD supervised analyses. MM carried out genetic simulations and pipeline automation, DGM performed information theoretic analyses. GAD and DGM drafted and all authors critically revised the manuscript.

\section{FUNDING}

347 This work was funded by NSF-EAR award \#1925535 to GD, NSF GRFP to MM, award \#61184 from the John Templeton Foundation to SIW. 
1. Antonelli, A. et al. Geological and climatic influences on mountain biodiversity. Nature 
23. Gutenkunst, R. N., Hernandez, R. D., Williamson, S. H. \& Bustamante, C. D. Inferring the Joint Demographic History of Multiple Populations from Multidimensional SNP Frequency Data. PLoS Genet 5, e1000695-11 (2009).

24. Mathew, L. A. \& Jensen, J. D. Evaluating the ability of the pairwise joint site frequency spectrum to co-estimate selection and demography. Front. Genet. 6, 268 (2015).

25. Haller, B. C. \& Messer, P. W. SLiM 3: Forward Genetic Simulations Beyond the WrightFisher Model. Molecular Biology and Evolution 36, 632-637 (2019).

26. De Mita, S. \& Siol, M. EggLib: Processing, analysis and simulation tools for population genetics and genomics. BMC Genetics 13, 27-12 (2012).

27. Finn, C. \& Lizier, J. T. Pointwise Partial Information Decomposition Using the Specificity and Ambiguity Lattices. Entropy 20, (2018).

28. Makkeh, A., Gutknecht, A. J. \& Wibral, M. A differentiable measure of pointwise shared information. arXiv [cs.IT] (2020).

29. Doane, D. P. Aesthetic Frequency Classifications. Am. Stat. 30, 181-183 (1976).

30. Scargle, J. D., Norris, J. P., Jackson, B. \& Chiang, J. Studies in astronomical time series analysis. Vi. Bayesian block representations. ApJ 764, 167 (2013).

31. Scott, D. W. Averaged Shifted Histograms: Effective Nonparametric Density Estimators in Several Dimensions. Ann. Stat. 13, 1024-1040 (1985). 
FIGURES

A

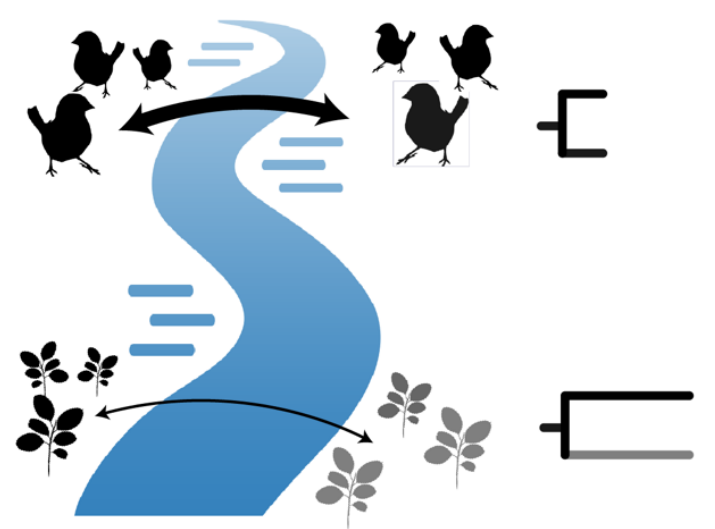

B

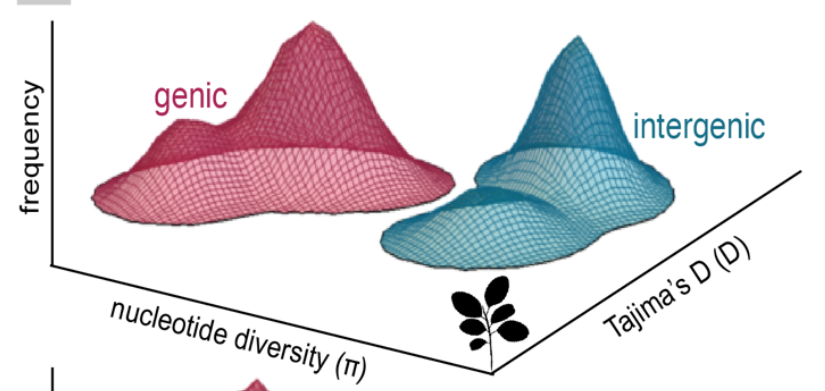

424

\section{5}

D

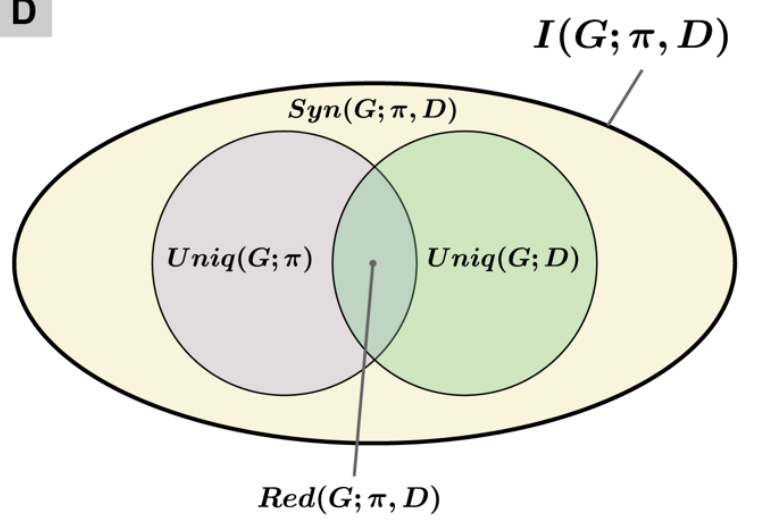

E

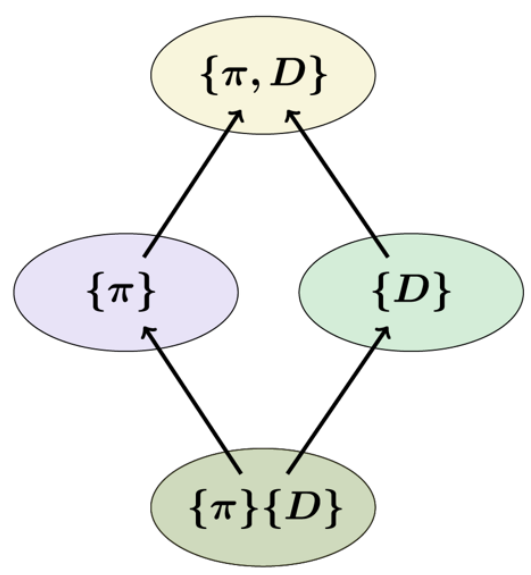

$\mathbf{F}$

$$
I(G ; A)=\sum_{\alpha \preccurlyeq\{A\}} \Pi_{R}(G ; \beta)
$$

$$
\begin{gathered}
I_{m i n}(G ; \alpha)=\sum_{g \in G} p(g) \min _{A_{i} \in \alpha}\left(\sum_{a \in A_{i}} p(a \mid g) \log \frac{p(g \mid a)}{p(g)}\right) \\
\Pi_{R}(G ; \alpha)=I_{m i n}(G ; \alpha)-\sum_{\beta \prec \alpha} \Pi_{R}(G ; \alpha)
\end{gathered}
$$

Figure 1 Conceptualization of partial information decomposition (PID) applied to genomic data (gPID). (A) Organisms may be affected differently by the same extrinsic events (e.g., formation of a river). (B) Genic and intergenic parts of the genome are differentially shaped by extrinsic (e.g., barrier to gene flow) and intrinsic (e.g., selection) forces that can be quantified with genetic metrics (e.g., $F_{S T}$, Tajima's D), the values of which will vary by taxon. (C) We calculated common metrics (m-nucleotide diversity, D- 
431 Tajima's $D$ ) in genic and intergenic regions of the genome on simulated data and (D,E) 432 calculated mutual information on these genetic statistics which is decomposed over a 433 lattice into unique, redundant, synergistic information terms given by (F). 


\section{A}

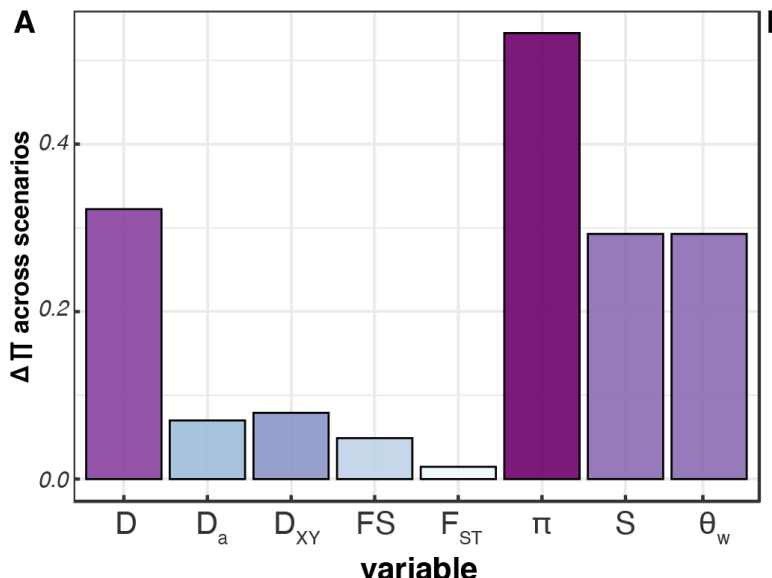

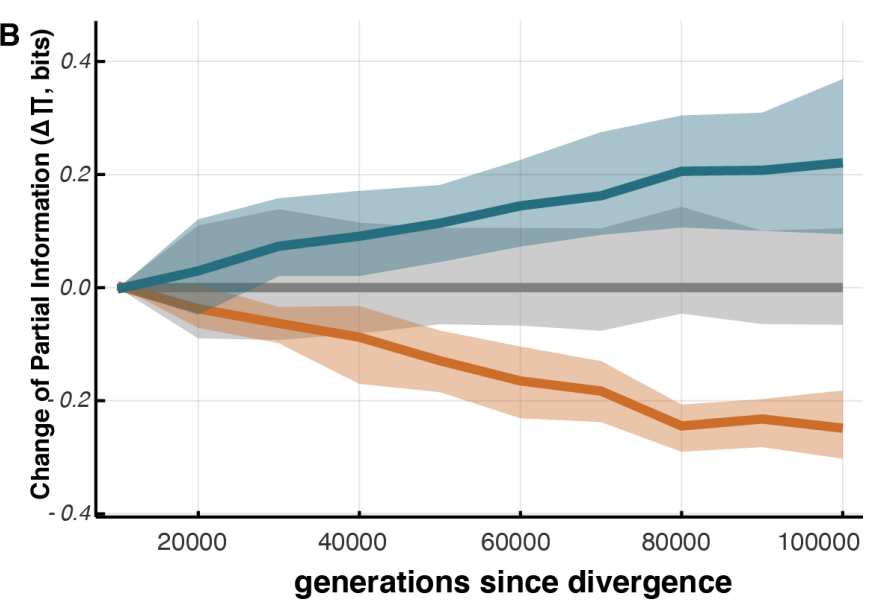

436 Figure 2. Summary of information content in genomic metrics. (A) Change in 437 information content $(\Delta \Pi)$ in single-variable decompositions across evolutionary 438 scenarios. A higher value reflects a greater ability for that variable to discriminate 439 among evolutionary scenarios. (B) Time series summary of how nodes from the 440 decomposed lattices change over time. Patterns were summarized into seven time441 signature clusters using k-means. Three of those are shown here: flat (grey, $442 \mathrm{~N}=303151$ ), increasing (turquoise, $\mathrm{N}=368$ ), and decreasing (orange, $\mathrm{N}=196$ ). 

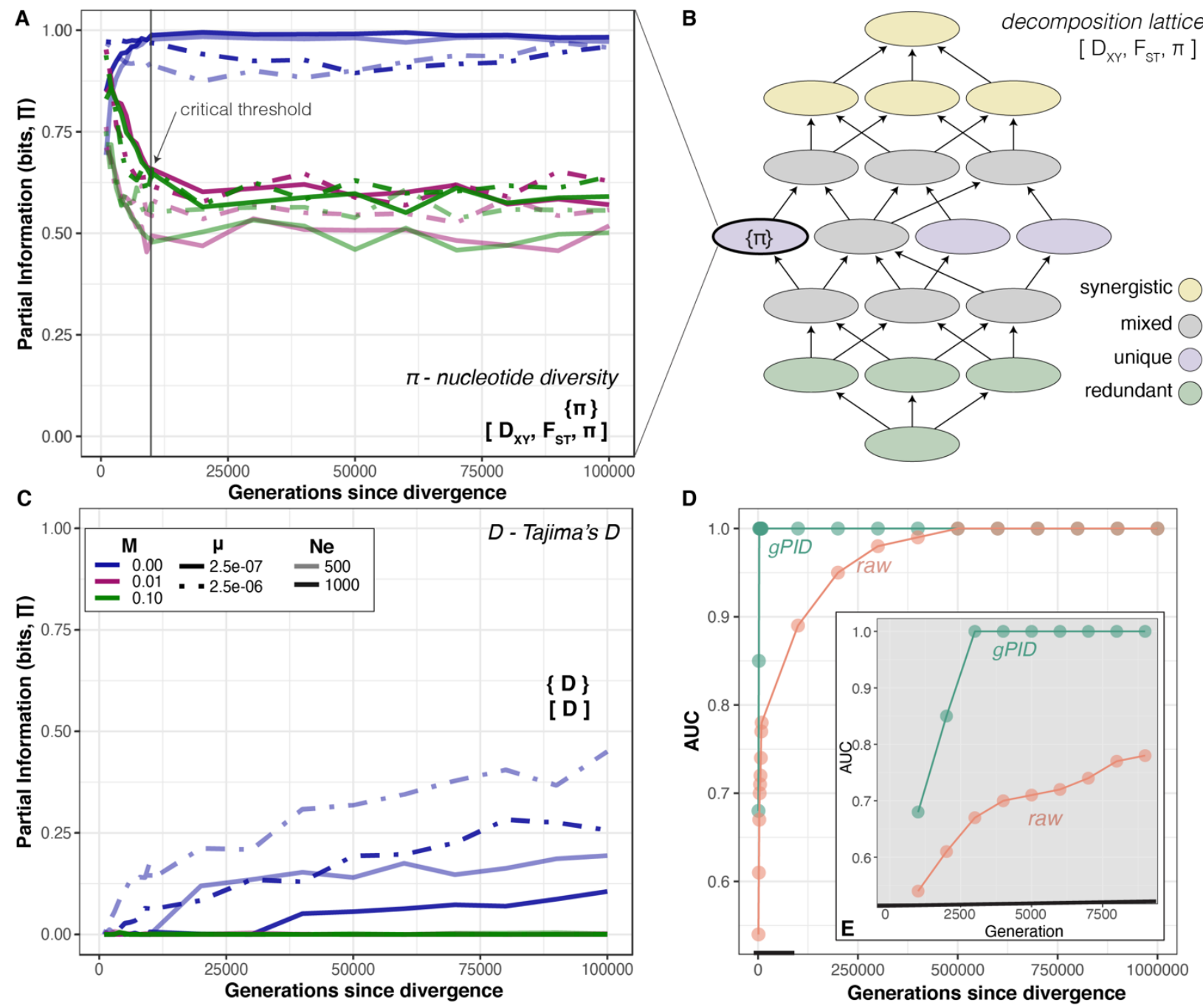

444 Figure 3. Discrimination of time-series signatures - (A) Partial information for 445 Unique\{Pi\} from [DXY, FsT, $\pi]$ over 100,000 generations, which shows the strongest 446 discrimination capability between gene flow (pink, green) and no gene flow (blue) 447 following a critical threshold when the pattern stabalizes. (B) The 18-node 448 decomposition lattice for [ $\left.D_{X Y}, F_{S T}, \pi\right]$, from which a single node $\{\pi\}$ is plotted in (a). 449 (C) Single variable decomposition of Tajima's D showing increasing partial information 450 for $\{D\}$ with time since divergence in the absence of gene flow, causing redistribution of 451 information across lattices. (D) Comparison of AUC values for a receiver-operator curve 452 (ROC) for the ability of $\pi$ to discriminate between presence/absence of gene flow using 453 gPID (green) versus raw $\pi$ values (orange). 
bioRxiv preprint doi: https://doi org/10.1101/2021.08.30.458276; this version posted September 1, 2021. The copyright holder for this preprint

(which was not certified by peer review) is the author/funder, who has granted bioRxiv a license to display the preprint in perpetuity. It is made available under aCC-BY-NC-ND 4.0 International license.
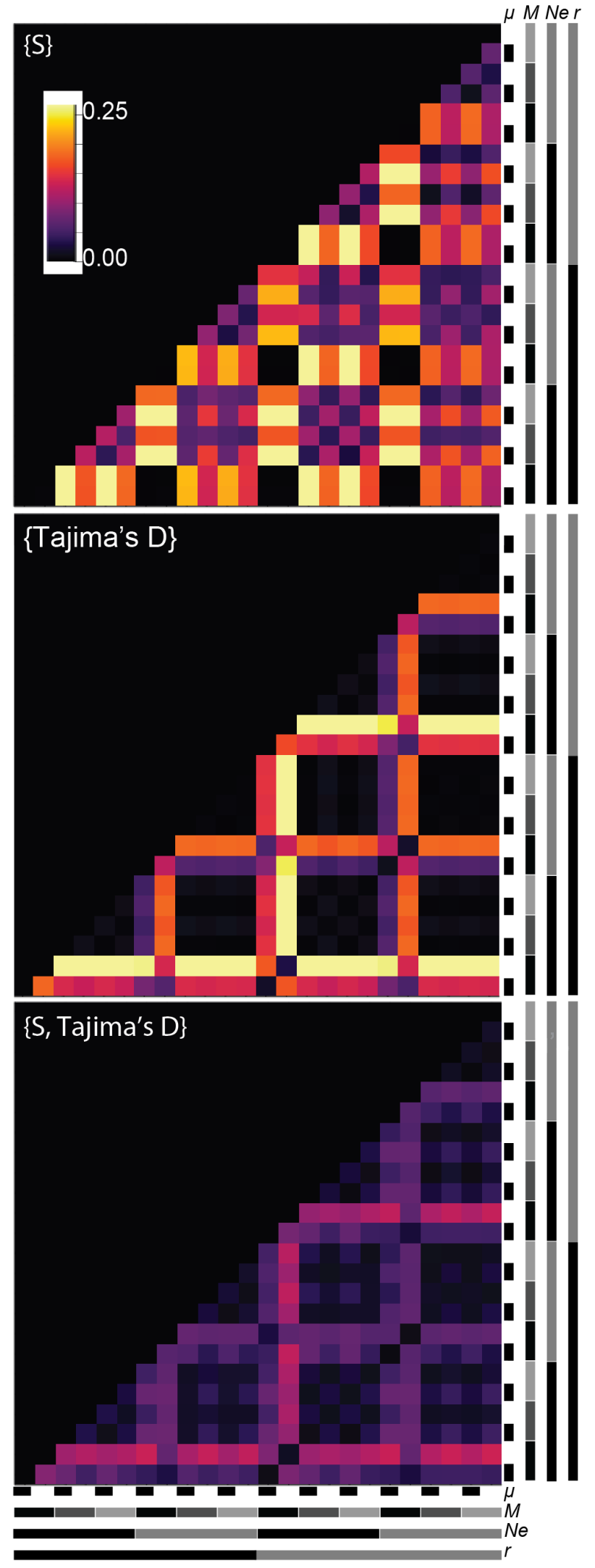
455 Figure 4. Additivity behavior of nodes in decomposed lattices. (A) Information 456 variability across evolutionary simulations for single decomposition of segregating sites 457 (S) and (B) Tajima's D. Higher values (yellow) indicate stronger discrimination ability. 458 Grey bars at bottom and right are guides for the heatmap pattern expected for each 459 variable simulated ( $\mu$ - mutation rate, $M$ - gene flow, $N_{e}$ - effective population size, $r$ 460 recombination rate). For example, Tajima's $D$ is discriminatory among gene flow 461 whereas $S$ detects both gene flow and mutation rate. (C) The information signatures are 462 additive, wherein $\{S$, Tajima's $D$ \} displays the additive effects from panels $A$ and $B$ and 463 the signal in heatmap (C) is exactly $1 / 4$ the sum of the signals in (A) and (B), 464 demonstrating true additivity. 